\title{
Natural polymorphisms in the resistance associated sites of HCV-G1 NS5B domain and correlation with geographic origin of $\mathrm{HCV}$ isolates
}

\author{
Sabrina Bagaglio ${ }^{1 *} \mathbb{D}$, Caterina Uberti-Foppa ${ }^{2}$, Alessandro Olgiati', Emanuela Messina ${ }^{1}$, Hamid Hasson', \\ Camilla Ferri $^{3}$ and Giulia Morsica ${ }^{1}$
}

\begin{abstract}
Background: We evaluated the frequency of naturally occurring resistance associated substitutions (RASs) and their characteristic of polymorphic or non-polymorphic amino acid change to direct acting antivirals (DAAs) in NS5b HCV subtypes $1 \mathrm{a}$ and $1 \mathrm{~b}$ according to different geographic origin of isolates.

Methods: Using a public database we retrieved 738 worldwide NS5b sequences (for which was available the geographic origin) from HCV genotype (G)1 infected patients naive to DAAs. NS5b sequences clustering with G1a were more conserved in regard of RASs than G1b isolates, (14\% vs 57\% RASs, $P<0.0001)$.

Results: In G1a, RASs were differently distributed between isolates from Europe (24\%) and USA, (12\%) $P=0.0186$. In particular, $421 \mathrm{~V}$ associated with resistance to non-nucleoside inhibitor beclabuvir was polymorphic in Europe and USA, being detected in $24 \%$ and $11 \%$ of sequences, respectively, $P=0.0140$.

In G1b, RASs were found in 45\% of sequences from Europe, in 54\% of isolates from USA and in $70 \%$ of sequences from Asia $(P=0.0051)$.

The $316 \mathrm{~N}$ polymorphism was detected in $54 \%$ of Asian isolates and at lower frequency, in $28 \%$ of isolates from USA and in $20 \%$ of European sequences $(P<0.0001)$.

Conclusions: In conclusion, a higher prevalence of RASs in G1b respect to G1a was found and a geographical distribution of RASs and polymorphic aa changes was observed in G1a as well in G1b.

The clinical and therapeutic impact of the geographic distribution of RASs to polymerase inhibitors remains to be established, particularly in patients with virologic failure to DAAs and/or advanced liver disease.
\end{abstract}

Keywords: Anti-HCV direct acting antivirals (DAAs), Geographic distribution, HCV genotype, NS5b polymerase inhibitors, Resistance associated substitutions (RASs)

\section{Background}

Among viral factors that may guide the treatment option the most important is the viral infecting genotype. The NS5b inhibitors are classified into nucleoside/nucleotide inhibitors $(\mathrm{Ni})$ and non-nucleoside inhibitors $(\mathrm{NNi})$. The $\mathrm{Ni}$ are considered active across all HCV genotypes, while $\mathrm{NNi}$ show restricted spectrum of activity against various

\footnotetext{
* Correspondence: bagaglio.sabrina@hsr.it

${ }^{1}$ Division of Infectious Diseases, Ospedale San Raffaele, Via Stamira d'Ancona 20, 20127 Milan, Italy

Full list of author information is available at the end of the article
}

genotypes, due to the rate of divergence of the binding site among different genotypes.

One other important viral factor is the genetic barrier to resistance related to the number and type of nucleotide substitutions required for the emergence of RASs during replication. The genetic barrier varies with drug class specificity over than HCV genotype and subtype [1].

In addition to the genetic barrier, the in vitro resistance level, corresponding to fold change under effective concentration (EC50) of a single compound in the replicon system, is commonly used to define the resistance profile of

(C) The Author(s). 2018 Open Access This article is distributed under the terms of the Creative Commons Attribution 4.0 International License (http://creativecommons.org/licenses/by/4.0/), which permits unrestricted use, distribution, and 
drug-selected variants [2-4]. The fitness of the variant under drug selection (replicative capacity of a resistant variant) may play also a crucial role in the virologic outcome [5].

Therefore, clinically relevant resistant strains are the result of a dynamic interaction between the fold change, the genetic barrier, and the replicative capacity of the virus selected during treatment with DAAs.

In this complex scenario, one important and debated possible virologic factor that may negatively influence the response to DAAs is the presence of RASs before any treatment with DAAs Baseline resistance testing is currently not routinely considered or recommended for initiating $\mathrm{HCV}$ treatment with DAAs, due to the overall high response (SVR) rates (> 90\%) [6-8].

However, pre-existing RASs in clinical strains have been shown to influence the virologic outcome for the combination of protease inhibitor asunaprevir with NS5a inhibitor daclatasvir [6] or for the combination of elbasvir/grazoprevir [7], or in patients with cirrhosis prior to daclatasvir/ sofosbuvir [8].

The studies on the impact of pre-existing NS5b RASs on the virologic outcome gave discordant results $[9,10]$.

For the NS3 protease gene, Pickett et al. [11] showed that HCV G1a isolates can be separated into at least two distinct clades, designated I and II. Several informative sites for this distinction are located within or proximal to codons associated with resistance to protease inhibitors or polymerase inhibitors. Santos and colleagues [12] showed that NS3 $80 \mathrm{~K}$ fell into a specific sub clade of G1a cladeI: NS3 $80 \mathrm{~K}$ polymorphism was associated with subclade IA, but nearly absent in subclades IB and IC. This finding providing an explanation for different resistance profiles to protease inhibitors of isolates clustering within cladeI.

This peculiar characteristic of HCV G1a suggests clade-specific differences that could modify the susceptibility of some clinical isolates to different classes of compounds.

A number of studies investigated the frequency of naturally occurring RASs into the NS5b domain of isolates retrieved from international data base [13, 14]. However, few data [15] are available on the presence of RASs within the NS5b domain according to geographic origin. Therefore, we decided to examine the frequency in isolates obtained from an international database, to better characterize genetic differences of HCV subtypes 1a and $1 \mathrm{~b}$ according to their geographic origin and distribution within clades.

\section{Methods}

The analysis considered only sequences of HCV G1a and G1b for whom was available the geographic origin. In total 1117 G1 NS5b sequences deposited before 2010 (to ensure that sequences were obtained from patients before exposure to DAAs activity) were obtained from Los Alamos $\mathrm{HCV}$ database, after exclusion of sequences containing stop codons in NS5b region, multiple sequences from the same patient and recombinant or clonal sequences. Of these 1117 isolates, 116 sequences were removed because G1 subtype was not indicated, 7 sequences clustering with G1 non-a non-b subtype were excluded, and 156 sequences for whom geographic origin was not available were also removed.

Thus, present study included 738 NS5b sequences: 432 clustered with G1a and 306 with G1b. Mutations analysis was performed at the resistance-associated positions for NS5b mutations already described in literature according to Ni or NNi compounds (Table 1).

According to previous report [12], amino acid changes were considered as polymorphic when present in at least

Table 1 Summary of substitutions associated with resistance to nucleoside and non-nucleoside NS5B inhibitors [26-28]

\begin{tabular}{|c|c|c|c|}
\hline Position & RAS & Genotype & Drug \\
\hline 159 & $\underline{F}$ & $1 a$ & SOF \\
\hline 237 & G & $1 a$ & SOF \\
\hline 282 & $\underline{\mathrm{R} / \mathrm{T}}$ & $1 a$ & SOF \\
\hline 316 & $\underline{F}$ & $1 a$ & SOF \\
\hline 320 & $\underline{F}$ & $1 a$ & SOF \\
\hline 321 & A & $1 a$ & SOF \\
\hline 159 & $\underline{F}$ & $1 b$ & SOF \\
\hline 282 & $\mathrm{G} / \mathrm{T}$ & $1 b$ & SOF \\
\hline 316 & $\mathrm{~F} / \mathrm{H} / \mathrm{N}$ & $1 b$ & SOF \\
\hline 321 & I & $1 b$ & SOF \\
\hline 314 & $\mathrm{H}$ & $1 a$ & DSV \\
\hline 316 & $\underline{Y}$ & $1 a$ & DSV \\
\hline 414 & $\underline{\mathrm{T} / \mathrm{V}}$ & $1 a$ & DSV \\
\hline 448 & $\underline{\mathrm{C} / \mathrm{H}}$ & $1 a$ & DSV \\
\hline 553 & IN & $1 a$ & DSV \\
\hline 554 & $\underline{s}$ & $1 a$ & DSV \\
\hline 556 & $\mathrm{G} / \mathrm{N} / \mathrm{R}$ & $1 a$ & DSV \\
\hline 558 & $\underline{R}$ & $1 a$ & DSV \\
\hline 559 & G & $1 a$ & DSV \\
\hline 316 & $\underline{\mathrm{Y} / \mathrm{H} / \mathrm{N}}$ & $1 \mathrm{~b}$ & DSV \\
\hline 368 & $\mathrm{~T}$ & $1 b$ & DSV \\
\hline 414 & ! & $1 \mathrm{~b}$ & DSV \\
\hline 445 & F & $1 b$ & DSV \\
\hline 448 & C & $1 \mathrm{~b}$ & DSV \\
\hline 553 & V & $1 b$ & DSV \\
\hline 556 & $\underline{\mathbf{G}}$ & $1 \mathrm{~b}$ & DSV \\
\hline 559 & $\underline{\mathrm{G} / \mathrm{N}}$ & $1 b$ & DSV \\
\hline 421 & $\underline{\mathbf{v}}$ & $1 a$ & $\mathrm{BCV}$ \\
\hline 495 & L/S & $1 a$ & $\mathrm{BCV}$ \\
\hline 499 & A/any & $1 b$ & DLV \\
\hline
\end{tabular}

$B C V$ beclabuvir, DSV dasabuvir, SOF sofosbuvir, DLV deleobuvir Amino acid substitutions detected in vivo in DAA failing patients are underlined, independently of in vitro data availability. Additionally, RAS detected only in vitro are indicated in bold 
$10 \%$ of isolates and non-polymorphic when detected in $<10 \%$ sequences.

\section{Statistical analysis}

Data were analyzed using Chi-Square or Fisher exact, when appropriate. A $P$-value $<0.05$ was considered statistically significant.

\section{Results}

Genotype distribution according to geographical origin of isolates

The geographic distribution of genotype 1 according to subtypes, showed that of 432 G1a sequences analyzed 63 isolates were from Europe, 368 from USA and only 1 sequence was from Asia. Of 306 G1b isolates, 69 sequences were from Europe, 137 from USA and 100 from Asia. The comparison of isolates from USA and Europe showed that sequences from USA belonged more frequently to G1a (73\% sequences) than to G1b (27\% sequences), while G1a (48\% sequences) and G1b (52\% sequences) were equally distributed in Europe, $P<0.0001$. Concerning the distribution of clades in G1a isolates, we found that clade I and II were differently distributed between isolates from USA and Europe. In isolates from USA, 280/368, (76\%) sequences belonged to cladeI, and the remaining 88 sequences to cladeII (24\%). In contrast, 19/63 (30\%) sequences from Europe clustered with cladeI and 44/63 (70\%) sequences with cladeII, $P<0.0001$.

\section{Global distribution of RASs in genotype 1}

In genotype $1,28 \%$ of sequences were mutated in sites of resistance to NS5b by population analysis. HCV NS5b sequences clustering with $\mathrm{HCV}$ G1a were more conserved in regard of RASs than HCV G1b isolates, $(60 / 432,13.8 \%$ vs.148/306, $48.3 \%$, mutated sequences, $P<0.0001)$.

\section{Geographic distribution of RASs and polymorphisms in genotype 1a}

In total, for G1a, RASs were detected in 60/432 (14\%) sequences. RASs were differently distributed between isolates from Europe $(15 / 63,24 \%)$ and USA (45/368, 12\%) $P=0.0186$. Only one isolate from Asia clustered with HCV genotype $1 \mathrm{a}$ and had not RASs.

Mutation $421 \mathrm{~V}$ with low level resistance $(\mathrm{FC}=3)$ to $\mathrm{NNi}$ beclabuvir, was more frequently detected in isolates from Europe $(15 / 63,24 \%)$ than in isolates from USA (42/ $368,11 \%) P=0.0140$. This aa change was polymorphic in Europe as well as in USA isolates (Fig. 1).

Despite this polymorphism was present in G1a cladeI as well as in G1a cladeII sequences from Europe and USA, a different distribution of RAS $421 \mathrm{~V}$ was observed in isolates belonging to G1a cladeII according to different geographic origin: this mutation was detected in 12/44 (27\%) G1-cladeII isolates from Europe, and in 2/88 (2\%) G1-cladeII isolates from USA, $P<0.0001$. None of analyzed sequences had RASs to deleobuvir.

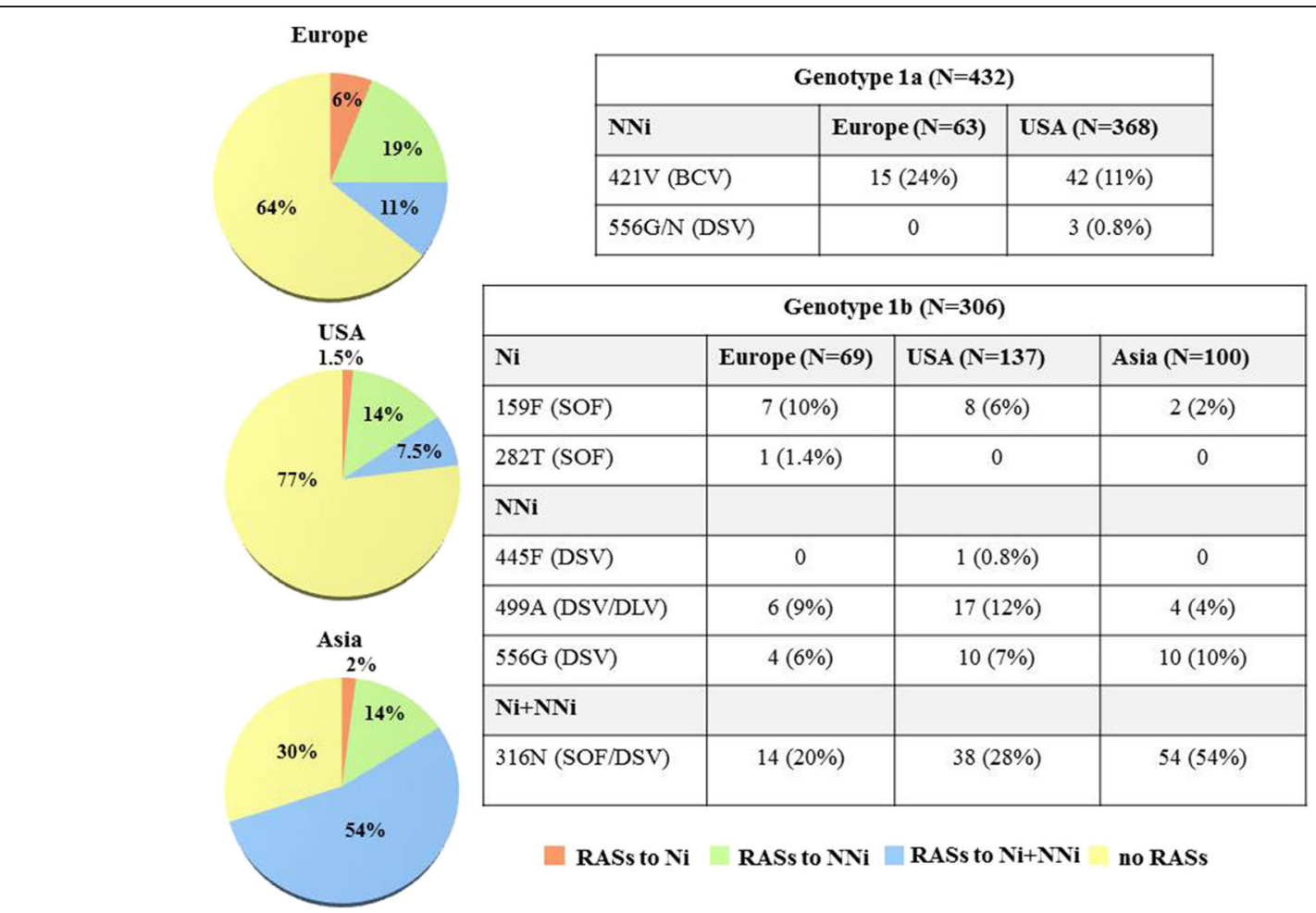

Fig. 1 Frequency and geographic distribution of RASs to NS5b Ni or NNi. BCV = beclabuvir, DSV= dasabuvir, SOF = sofosbuvir, DLV= deleobuvir 
None of RASs described for sofosbuvir was detected, while 3 mutated sequences at position $556 \mathrm{G} / \mathrm{N}(\mathrm{FC}=30)$ and associated with resistance to $\mathrm{NNi}$ dasabuvir were found, all among USA isolates. Fold change for each RAS and replicative capacity of G1a resistance mutations are depicted in Table 2.

\section{Geographic distribution of RASs and polymorphisms in genotype $1 \mathrm{~b}$}

A high prevalence of NS5b RASs was detected in G1b (176/306; 57\% sequences were mutated at sites associated with resistance). Considering all sequences clustering with G1b, a different distribution of RASs according to geographic origin, was found. RASs were detected in $32 / 69$ (45\%) sequences from Europe, in 74/137 (54\%) isolates from USA and in 70/100 (70\%) sequences from Asia $(P=0.0051)$. The distribution of RASs to $\mathrm{Ni}$ vs $\mathrm{NNi}$ according to geographic origin is described in Fig. 1.

In detail, the $159 \mathrm{~F}$ ( $\mathrm{FC}=1.3$ to $\mathrm{Ni}$ sofosbuvir) was present in $7 / 69$ (10.1\%) European sequences, in 8/137 (5.8\%) sequences from USA and in 2/100 (2\%) Asian sequences, showing a trend toward significance, $P=0.0743$ (Fig. 1).

The RAS $316 \mathrm{~N}$, (FC $=1.6$ to Ni sofosbuvir, and $\mathrm{FC}=5$ to $\mathrm{NNi}$ dasabuvir) was more frequently detected in Asian isolates $(54 / 100,54 \%)$ respect to isolates from USA (38/136; $28 \%)$ and Europe $(14 / 69 ; 20 \%), P<0.0001$. The RAS $316 \mathrm{~N}$ was polymorphic in Asia as well as in Europe and USA, being present in $54 \%$ Asian isolates and in $>10 \%$ of sequences from Europe or USA (Fig. 1). The RASs 159F and $316 \mathrm{~N}$ were concomitantly detected in $2 \%$ isolates from Asia, $5 \%$ of isolates from USA and 10\% isolates from Europe.

Twenty-seven/306 (8.8\%) sequences harbored the RAS 499A ( $\mathrm{FC}=6$ to $\mathrm{NNi}$ deleobuvir; $\mathrm{FC}=1.4$ to $\mathrm{NNi}$ dasabuvir): 17/137 (12.4\%), were from USA, $6 / 69$ (8.6\%) from Europe and 4/100 (4\%) from Asia, this result showing a trend towards significance, $P=0.0788$. So, on the basis of data retrieved from Los Alamos, the 499A substitution seemed polymorphic in USA but not in Europe and Asia. The 556G substitution ( $\mathrm{FC}=11$ to dasabuvir) was similarly distributed among isolates from Asia, USA, and Europe, being detected in 10\% (10/100) of Asian isolates, $7 \%(10 / 137)$ of isolates from USA, 6\% (4/69) of European sequences, $P=0.577$ (Fig. 1). One sequence from Europe had $282 \mathrm{~T}$ ( $\mathrm{FC}=10$ to $\mathrm{Ni}$ sofosbuvir) and 1 sequence from USA had $445 \mathrm{~F}$ ( $\mathrm{FC}=16$ to dasabuvir). Finally, the $316 \mathrm{~N}$ associated with 556G was detected in 4/ $137(3 \%)$ isolates from USA, in 3/69 (4\%) isolates from Europe and in 3 (3\%) sequences from Asia. Fold change of each RAS and replicative capacity of G1b resistance mutations are shown in Table 2.

\section{Discussion}

In the present study, we observed a higher prevalence of RASs in G1b respect to G1a and a geographical distribution of RASs as well as polymorphic aa change in G1a and G1b, by retrieving sequences from an international data base, Concerning G1a sequence analysis, we showed that the aa substitution $421 \mathrm{~V}$ was polymorphic (detected in $>10 \%$ of sequences) in G1a isolates from USA and Europe.

In the study by Patino-Galindo et al. [13], evaluating global prevalence of natural RASs to polymerase inhibitors, $421 \mathrm{~V}$ was detected in $12.56 \%$ of G1a sequences; however, the geographic origin of polymorphisms was not reported.

Recently, some reports [12, 16, 17], investigated the geographic distribution of polymorphism NS3 $80 \mathrm{~K}$ and its segregation into a specific clade, providing explanation for different resistance profiles to protease inhibitors of isolates from different geographical area and within different clades. Interestingly, we found that the isolates from Europe harboring NS5b $421 \mathrm{~V}$ polymorphism fell more frequently in G1a cladeII, while $421 \mathrm{~V}$ polymorphism in USA clustered more

Table 2 Fold change and replicative capacity of naturally occurring RASs in isolates retrieved from Los Alamos HCV database

\begin{tabular}{|c|c|c|c|c|c|}
\hline RAS & $\mathrm{DRUG}^{\mathrm{a}}$ & HCV subtype & Fold change & Replicative capacity & References \\
\hline $421 \mathrm{~V}$ & $\mathrm{BCV}$ & $1 \mathrm{a}$ & 3 & $N A^{b}$ & - \\
\hline $556 G$ & DSV & $1 \mathrm{a}$ & 30 & $59 \%$ & {$[2]$} \\
\hline $556 \mathrm{~N}$ & DSV & $1 a$ & 29 & NA & - \\
\hline $282 \mathrm{~T}$ & SOF & $1 b$ & 10 & $5 \%$ & {$[3,4]$} \\
\hline $159 F$ & SOF & $1 b$ & 1.3 & $25 \%$ & {$[3,4]$} \\
\hline \multirow[t]{2}{*}{$316 N$} & SOF & $1 b$ & 1.6 & $154 \%$ & [2] \\
\hline & DSV & & 5 & $154 \%$ & [2] \\
\hline $556 \mathrm{G}$ & DSV & $1 b$ & 11 & $62 \%$ & [2] \\
\hline $445 \mathrm{~F}$ & DSV & $1 b$ & 16 & NA & - \\
\hline \multirow[t]{2}{*}{$499 \mathrm{~A}$} & DLV & $1 b$ & 6 & NA & - \\
\hline & DSV & $1 b$ & 1.4 & NA & - \\
\hline
\end{tabular}

${ }^{\mathrm{a}} B C V$ beclabuvir, $D S V$ dasabuvir, SOF sofosbuvir, $D L V$ deleobuvir ${ }^{\mathrm{b}} N A$ not available 
frequently within G1a cladeI. The majority of $421 \mathrm{~V}$ G1a cladeII mutated sequences detected in Europe were from Switzerland. Therefore, it is possible that this polymorphic site within G1a cladeII is a peculiar characteristic of isolates from this country rather than a common characteristic of European strains.

Unfortunately, in the present study no additional sequences from other countries in Europe were available to draw conclusions on the distribution of $421 \mathrm{~V}$ polymorphism into different clades (I or II) according to geographical origin of isolates.

In line with previous studies $[13,15]$, mutation $556 \mathrm{G}$ (RAS to NNi dasabuvir but not to NNi deleobuvir and $\mathrm{Ni}$ sofosbuvir) was rarely detected in G1a $(0.6 \%$ of isolates).

Concerning the G1b isolates, we globally detected 5\% of $159 \mathrm{~F}$ substitution. By population analysis, the $159 \mathrm{~F}$ was polymorphic in Europe, but not in Asia and USA. This data is in accordance with that of Patino-Galindo et al. [13] and Welzel et al. [15], indicating that the 159F was present respectively in $4.5 \%$ or $8 \%$ of isolates obtained from an international database .

In our study, the frequency of $159 \mathrm{~F}$ was similar to that described in a previous study [4], exploring the baseline resistance profile of isolates obtained from sofosbuvir (SOF) or ledipasvir (LDV) plus sof trials and showing that the $159 \mathrm{~F}$ was present by deep sequencing cut off $15 \%$, in $7 \%$ G1b patients. In this report was also investigated the presence of baseline RASs for polymerase inhibitors in individuals with virologic failure, showing that $2 / 6$ patients harboring the mutation $159 \mathrm{~F}$ in sof trials and $0 / 23$ in SOF/LDV trials, experienced virologic failure. The authors concluded that baseline 159F was not associated with treatment failure because the rate of treatment response in these two studies were similar in individuals with or without 159F.

In another study, Isakov et al. [18], showed that, among G1b infected patients, the presence of $159 \mathrm{~F}$ at baseline was associated with a lower SVR rate in patients treated for 16 weeks, respect to those treated for 24 weeks.

In liver pre-transplant studies [18], 4/4 G1b infected patients with pretreatment $159 \mathrm{~F}$ as dominant variant (> $99 \%$ within the viral population) experienced virologic failure. Considering aforementioned studies, it is possible that not only the duration of the regimen including a single DAA and/or the presence of advanced liver disease, but also the proportion of quasispecies harboring $159 \mathrm{~F}$ polymorphism at baseline, have contributed to the failure. In the majority of reports $[9,14,20]$ evaluating natural RASs to polymerase inhibitors, the $316 \mathrm{~N}$ associated with resistance to dasabuvir and sofosbuvir was detected in $10-36 \%$ of G1b isolates. We found the aa substitution $316 \mathrm{~N}$ in $35 \%$ of G1b sequences, with higher frequency in Asian isolates compared to those from Europe or USA.

In contrast, the study by Chen et al. [19] showed that, none of 361 sequence of genotype $1 \mathrm{~b}$ harbored $316 \mathrm{~N}$, despite isolates from Asia were also included in their analysis. According to previous studies performed by means of population sequencing in clinical strains from Asia, that did not found [21], or found at very low percentage [22] the $159 \mathrm{~F}$ associated with $316 \mathrm{~N}$, these two mutations were concomitantly present in only two isolates coming from Japan, despite the high frequency of $316 \mathrm{~N}$ in this area. However, in the study by Ito et al. [22], the deep sequencing analysis of clinical strains demonstrated a high frequency (30\%) of $159 \mathrm{~F}$ among patients harboring $316 \mathrm{~N}$ polymorphism. In the present study, we showed that a high number of isolates in Europe and USA naturally harbored $316 \mathrm{~N}$ in combination with 159F. In this regard, the aa substitution $316 \mathrm{~N}$ has been described as a compensatory mutation after the selection of the $159 \mathrm{~F}$ mutation, giving a selective advantage of the variant with concomitant $159 \mathrm{~F}$ and $316 \mathrm{~N}$. In vitro studies $[3,4]$ showed that the replication capacity of $159 \mathrm{~F}$ is relatively low, $0.24-0.25$ fold compared with the wild-type virus, while in the case with the co-existence of the $159 \mathrm{~F}$ and the $316 \mathrm{~N}$ substitutions, the replication capacity is $0.65-1.14$ fold respect to the wild-type virus. Therefore, the higher replication capacity of the double mutant respect to the $159 \mathrm{~F}$ variant and to the wild type, may confer a higher level of resistance than $159 \mathrm{~F}$ alone to $\mathrm{Ni}$ sofosbuvir. Unfortunately, the presence of this double mutant was not evaluated in the study by Welzel et al. [15], exploring a large number of sequences around the world and coming from clinical trials. So, no additional information on its frequency in global population is available so far.

In this context, Donaldson et al. [23], using bioinformatics analysis, showed that the cysteine to asparagine change at NS5b position 361 introduces a larger aa than the C316 and that the larger amino acid is predicted to interfere with the ability of sofosbuvir to enter the active site, providing molecular basis for its potential role in the resistance pattern to Ni sofosbuvir.

In G1b, RAS 499A (RAS to NNi deleobuvir and dasabuvir) was polymorphic in USA but not in Europe and Asia. This RAS was previously detected in $16.8 \%$ of patients from China [21]. In one other study [13] 499A was detected in $9.8 \%$ of G $1 \mathrm{~b}$ isolates. However no information was provided on the geographic origin of this RAS.

According to previous report [13], the S556G was more frequently detected in G1b respect to G1a (7\% vs. $0.6 \%$ of frequency, respectively). One study from China [22] showed $3.7 \%$ of $556 \mathrm{G} / \mathrm{N}$ in G1b infected patients. In two other studies $[9,19] 556 \mathrm{~N}$ was present in $9.6 \%$ 
and $7 \% 1 \mathrm{~b}$ isolates, respectively and in $1.4 \%$ and $0.6 \% 1 \mathrm{a}$ isolates, respectively.

In one recent clinical trial [24] evaluating the efficacy of 8 weeks treatment with ombitasvir /paritaprevir /ritonavir/dasabuvir, in G1b infected non cirrhotic patients, 2 patients who experienced relapse, harbored NS5b $316 \mathrm{~N}$ and NS5b 556G at baseline and at the time of relapse. In such cases natural polymorphisms in sites of resistance might affect treatment outcome. In regard of this issue, one clinical study [25] of re-treatment with polymerase inhibitors-containing regimens, showed that $159 \mathrm{~F}$ associated or not with $282 \mathrm{~T}$ was more frequently selected when patients previously exposed to SOF/LDV for 8 or 12 weeks, were re-treated with the same regimen for 24 weeks (2/12 virologic failure among 41 re-treated patients, had $159 \mathrm{~F}$ alone or associated with $282 \mathrm{~T}$ ).

Profiling of geographic distribution for polymorphic aa changes could add important information for the future treatment strategies; the mutational profile of NS5b polymerase region according to different HCV subtypes and their geographic origin, can be important especially in difficult-to-cure patients for whom new treatment strategies could involve complex association regimens. Viruses carrying polymorphisms and in particular those harboring two or more RASs may attenuate $\mathrm{Ni}$ and $\mathrm{NNi}$ activity affecting future treatment options.

\section{Abbreviations}

aa: Amino acid; BCV: Beclabuvir; DAAs : Direct acting antivirals; DLV: Deleobuvir; DSV: Dasabuvir; EC50: Effective concentration 50; FC: Fold change; G: Genotype; HCV: Hepatitis C virus; LDV: Ledipasvir; Ni: Nucleoside/ nucleotide inhibitor; NNi: Non-nucleoside inhibitor; RAS: Resistance associated substitutions; SOF: Sofosbuvir

\section{Funding}

No funding was used in the preparation of this manuscript.

\section{Availability of data and materials}

Sequences analyzed were available on website of Los Alamos HCV data base (https://hcv.lanl.gov). Criteria applied for sequences selection were described in methods section.

\section{Authors' contributions}

SB and GM conceived and designed the experiments; $A O$ and EM analyzed the data; CUF, CF and HH contributed analysis tools; SB and GM wrote the paper. All authors read and approved the final manuscript.

\section{Ethics approval and consent to participate}

Not applicable.

\section{Consent for publication}

Not applicable.

\section{Competing interests}

The authors declare that they have no competing interests.

\section{Publisher's Note}

Springer Nature remains neutral with regard to jurisdictional claims in published maps and institutional affiliations.

\section{Author details}

'Division of Infectious Diseases, Ospedale San Raffaele, Via Stamira d'Ancona 20, 20127 Milan, Italy. ${ }^{2}$ Vita Salute University, Milan, Italy. ${ }^{3}$ Pharmacy,

Ospedale San Raffaele, Milan, Italy.

Received: 6 February 2018 Accepted: 10 September 2018

Published online: 18 September 2018

\section{References}

1. Powdrill MH, Tchesnokov EP, Kozak RA, Russell RS, Martin R, Svarovskaia ES, et al. Contribution of a mutational bias in hepatitis $C$ virus replication to the genetic barrier in the development of drug resistance. Proc Natl Acad Sci U S A. 2011;108: 20509-13.

2. Kati W, Koev G, Irvin M, Beyer J, Liu Y, Krishnan P, et al. In vitro activity and resistance profile of dasabuvir, a nonnucleoside hepatitis $C$ virus polymerase inhibitor. Antimicrob Agents Chemother. 2015;59:1505-11.

3. Liu M, Tuttle M, Gao M, Lemm JA. Potency and resistance analysis of hepatitis C virus NS5B polymerase inhibitor BMS-791325 on all major genotypes. Antimicrob Agents Chemother. 2014;58:7416-23.

4. Svarovskaia ES, Gane E, Dvory-Sobol H, Martin R, Doehle B, Hedskog C, et al. L159F and V321A Sofosbuvir-associated hepatitis C virus NS5B substitutions. J Infect Dis. 2016:213:5-7.

5. Pawlotsky JM. Hepatitis $C$ virus resistance to direct-acting antiviral drugs in interferon-free regimens. Gastroenterology. 2016:151:70-86.

6. Manns M, Pol S, Jacobson IM, Marcellin P, Gordon SC, Peng CY, et al. All-oral daclatasvir plus asunaprevir for hepatitis $C$ virus genotype $1 \mathrm{~b}$ : a multinational, phase 3, multicohort study. Lancet. 2014;384:1597-605.

7. Zeuzem S, Ghalib R, Reddy KR, Pockros PJ, Ben Ari Z, Zhao Y, et al. Grazoprevir- elbasvir combination therapy for treatment-naive cirrhotic and non cirrhotic patients with chronic hepatitis C virus G1, 4, or 6 infection: a randomized trial. Ann Intern Med. 2015;163(1):13.

8. DaklinzaTM. Bristol-Myers Squibb Company, Princeton, NJ; 2016. Available from: http://packageinserts.bms.com/pi/pi_daklinza.pdf.

9. Krishnan P, Tripathi R, Schnell G, Reisch T, Beyer J, Irvin M, et al. Resistance analysis of baseline and treatment-emergent variants in hepatitis $C$ virus genotype 1 in the AVIATOR study with paritaprevir-ritonavir, ombitasvir, and dasabuvir. Antimicrob Agents Chemother. 2015:59:5445-54.

10. Zeuzem S, Mizokami M, Pianko S, Mangia A, Han KH, Martin R, et al. NS5A resistance-associated substitutions in patients with genotype 1 hepatitis $C$ virus: prevalence and effect on treatment outcome. J Hepatol. 2017;66:910-8.

11. Pickett BE, Striker R, Lefkowitz EJ. Evidence for separation of HCV subtype 1a into two distinct clades. J Viral Hepat. 2011;18:608-18.

12. Santos AF, Bello G, Vidal LL, Souza SL, Mir D, Soares MA. In-depth phylogenetic analysis of hepatitis $C$ virus subtype 1a and occurrence of 80K and associated polymorphisms in the NS3 protease. Sci Rep. 2016;6:31780.

13. Patiño-Galindo JÁ, Salvatierra K, González-Candelas F, López-Labrador FX. Comprehensive screening for naturally occurring hepatitis $C$ virus resistance to direct-acting antivirals in the NS3, NS5A, and NS5B genes in worldwide isolates of viral genotypes 1 to 6. Antimicrob Agents Chemother. 2016;60:2402-16.

14. Alves R, Queiroz ATL, Pessoa MG, da Silva EF, Mazo DFC, Carrilho FJ, Carvalho-Filho RJ, de Carvalho IMVG. The presence of resistance mutations to protease and polymerase inhibitors in hepatitis $C$ virus sequences from the Los Alamos databank. J Viral Hep. 2013;20:414-21.

15. Welzel TM, Bhardwaj N, Hedskog C, Chodavarapu K, Camus G, McNally J, et al. Global epidemiology of HCV subtypes and resistance-associated substitutions evaluated by sequencing-based subtype analyses. J Hepatol 2017; pii: S0168-8278.

16. De Luca A, Di Giambenedetto S, Lo Presti A, Sierra S, Prosperi M, Cella E, et al. Two distinct hepatitis C virus genotype 1a clades have different geographical distribution and association with natural resistance to NS3 protease inhibitors. Open Forum Infect Dis. 2015;2:ofv043.

17. Sarrazin C, Lathouwers E, Peeters M, Daems B, Buelens A, Witek J, Wyckmans Y, Fevery B, Verbinnen T, Ghys A, Schlag M, Baldini A, De Meyer $\mathrm{S}$, Lenz O. Prevalence of the hepatitis C virus NS3 polymorphism Q80K in genotype 1 patients in the European region. Antivir Res. 2015;116:10-6.

18. Isakov V, Zhdanov K, Kersey K, Svarovskaia E, Massetto B, Zhu Y, et al. Efficacy of sofosbuvir plus ribavirin in treatment-naive patients with genotype-1 and -3 HCV infection: results from a Russian phase IIIb study. Antivir Ther. 2016:21:671-8.

19. Chen ZW, Li H, Ren H, Hu P. Global prevalence of pre-existing HCV variants resistant to direct-acting antiviral agents (DAAs): mining the GenBank HCV genome data. Sci Rep. 2016;b6:203-10. 
20. Sarrazin C. The importance of resistance to direct antiviral drugs in HCV infection in clinical practice. J Hepatol. 2016;64:486-504.

21. Wang Y, Rao HY, Xie XW, Wei L. Direct-acting Antiviral Agents Resistanceassociated Polymorphisms in Chinese Treatment-naïve Patients Infected with Genotype 1b Hepatitis C Virus. Chin Med J. 2015;128:2625-31.

22. Ito J, Suda G, Yamamoto Y, Nagasaka A, Furuya K, Kumagai K, et al. Prevalence and characteristics of naturally occurring sofosbuvir resistanceassociated variants in patients with hepatitis C virus G1b infection. Hepatol Res. 2016:46:1294-303.

23. Donaldson EF, Harrington PR, O'Rear JJ, Naeger LK. Clinical evidence and bioinformatics characterization of potential hepatitis $\mathrm{C}$ virus resistance pathways for sofosbuvir. Hepatology. 2015;61:56-65.

24. Welzel TM, Asselah T, Dumas EO, Zeuzem S, Shaw D, Hazzan R, et al. Ombitasvir, paritaprevir, and ritonavir plus dasabuvir for 8 weeks in previously untreated patients with hepatitis $C$ virus genotype $1 \mathrm{~b}$ infection without cirrhosis (GARNET): a single-arm, open-label, phase 3b trial. Lancet Gastroenterol Hepatol. 2017;2:494-500.

25. Lawitz E, Flamm S, Yang JC, Pang PS, Zhu Y, Svarovskaia E, et al. Retreatment of patients who failed 8 or 12 weeks of ledipasvir/sofosbuvirbased regimens with ledipasvir/sofosbuvir for 24 weeks. Journal of Hepato 2015; 62: S192. Abstract number O005.

26. Sorbo MC, Cento V, Di MVC, Howe AYM, Garcia F, Perno CF, Francesca Ceccherini-Silberstein F. Hepatitis C virus drug resistance associated substitutions and their clinical relevance. Update 2018 Drug Resistance Updates. 2018;37:17-39.

27. Berger KL, Sarrazin C, Nelson DR, Scherer J, Sha N, Marquis M, Côté-Martin A Vinisko R, Stern JO, Mensa FJ, George Kukolj G. Resistance analyses of HCV NS3/4A protease and NS5B polymerase from clinical studies of Deleobuvir and Faldaprevir. PLoS One. 2016;11:e0160668.

28. Rigat KL, Lu H, Wang YK, Argyrou A, Fanslau C, Beno B, Wang Y, Marcinkeviciene J, Ding M, Gentles RG, Gao M, Abell LM, Roberts SB, Mechanism of inhibition for BMS-791325, a novel non-nucleoside inhibitor of hepatitis C virus NS5B polymerase. J Biol Chem. 2014;289:33456-68.

Ready to submit your research? Choose BMC and benefit from:

- fast, convenient online submission

- thorough peer review by experienced researchers in your field

- rapid publication on acceptance

- support for research data, including large and complex data types

- gold Open Access which fosters wider collaboration and increased citations

- maximum visibility for your research: over $100 \mathrm{M}$ website views per year

At $\mathrm{BMC}$, research is always in progress.

Learn more biomedcentral.com/submissions 